This manuscript has been accepted for publication in The Lancet Group. The manuscript will undergo copyediting, typesetting, and review of the resulting proof before it is published in its final form. Please note that during the production process errors may be discovered which could affect the content, and all disclaimers that apply to the journal apply to this manuscript. A definitive version was subsequently published in Lancet Psychiatry, [VOL 3, ISSUE 6, (June 1, 2016)] DOI http://dx.doi.org/10.1016/S22150366(16)30040-2

\title{
When Can Neurofeedback Join the Clinical
}

\section{Armamentarium?}

\author{
Robert T. Thibault ${ }^{\mathrm{a}}$ and Amir Raz ${ }^{\mathrm{a}, \mathrm{b}, \mathrm{c}}$
}

${ }^{a}$ McGill University, 3775 University Street, Montreal, QC, H3A 2B4, Canada

${ }^{\mathrm{b}}$ The Lady Davis Institute for Medical Research at the Jewish General Hospital, 3755 Cote Ste.

Catherine, Montreal, QC, H3T 1E2, Canada

${ }^{\mathrm{c}}$ Institute for Community and Family Psychiatry, 4333 Cote Ste. Catherine, Montreal, QC, H3T

1E4, Canada

*Please address correspondence to:

Professor Amir Raz, 4333 Cote Ste. Catherine, Montreal, QC, H3T 1E4, Canada

amir.raz@mcgill.ca

Tel: 1-514-340-8210; Fax: 1-514-340-8124

First author email: robert.thibault@mail.mcgill.ca 
Neurofeedback appears to both improve normal brain function ${ }^{1}$ and treat a wide range of mental disorders, including attention deficit hyperactivity disorder (ADHD), epilepsy, depression, anxiety, insomnia, autism spectrum disorder, and alcoholism. ${ }^{2}$ Despite a relatively long history, however, the medical community continues to question the clinical utility of this technique. To earn widespread appellation as evidence-based medicine, neurofeedback must meet three challenges: 1) perform at least on par with standard-of-care treatments in randomized controlled trials for each disorder where neurofeedback purports to help; 2) consistently outperform highly comparable placebo control conditions (e.g., sham neurofeedback); and 3) establish a clear mechanism for the claimed therapeutic benefits.

In electroencephalographic (EEG) neurofeedback - the earliest and most widely practised form of neurofeedback ${ }^{3}$ - participants attempt to modulate an on-going feedback signal derived from real-time electrical activity of their own brain. In learning to control a particular brain signal, participants allegedly improve an associated behaviour. The underlying brain-based theory of this neurofeedback dynamic draws on research correlating clinical disorders with quantitative differences in EEG signal, yet rests on an unsupported tendency to reduce complex overarching behaviours to circumscribed brain processes. Relevant studies, moreover, seldom demonstrate that receiving neurofeedback, let alone a precise brain signal, constitutes a necessary component to attain the supposed benefits. ${ }^{3}$ Alternatively, psychosocial factors (e.g., expectation and motivation), rather than neurophysiological parameters, may mediate the reported clinical improvement. Typical EEG-neurofeedback protocols require participants to visit a medical clinic for 20-40 sessions and interface with seemingly cutting-edge brain technology. ${ }^{3}$ Future research should tease apart and examine these quantifiable psychosocial factors (e.g., time spent at clinic, 
confidence in neurofeedback technology) to allow a better scientific understanding of how and to what degree such influences drive the measured outcomes.

Few consumers and practitioners appreciate that EEG-neurofeedback helps patients regardless of the feedback source. ${ }^{3}$ In other words, sham neurofeedback (e.g., from irrelevant brain activity or even from a different brain) improves treatment outcomes as much as true neurofeedback. ${ }^{3,4}$ After a thorough literature search (query "neurofeedback OR biofeedback AND electroenchapalogra* OR EEG" in Scopus ${ }^{\circledR}$, Web of Science ${ }^{\mathrm{TM}}$, and Google Scholar) we could find only one sham-controlled, double-blind EEG neurofeedback study that demonstrated clinical superiority of veridical over sham feedback. ${ }^{5}$ This study engaged 32 chronic stroke patients and found that, in conjunction with physiotherapy, participants who received genuine brain-based feedback, compared to random feedback, better improved motor control of their affected arm (3.41 versus 0.35 points on the 54-point upper limb Fugl-Meyer motor score assessment, $\mathrm{p}=0.018) .{ }^{5}$ The other clinical EEG-neurofeedback studies featured either inadequate experimental design (e.g., to disentangle brain-based mechanisms from psychosocial influences) or comparable effects between real and sham feedback. Subsequently, placebo factors likely account for most research findings and clinical improvements related to EEGneurofeedback. ${ }^{3}$ While contemporary biomedicine often dismisses placebo outcomes as "noise" or "non-effects", many standard treatments benefit from placebos. ${ }^{6}$ Future research should explore the healing mechanisms common to true and sham neurofeedback, including the role of motivation, expectation, interaction with health professionals, and demand characteristics. 
Despite much research on the clinical benefits of EEG-neurofeedback ${ }^{7}$, only a few studies - all addressing paediatric ADHD - tested a direct comparison of neurofeedback to currently accepted treatments. ${ }^{8-11}$ Two of these experiments reported comparable improvements in attention between the neurofeedback and medication groups yet shied away from collecting neurological measures, ${ }^{8,9}$ one showed similar changes in resting-state EEG activity yet neglected to ascertain whether attention actually improved $;{ }^{10}$ the other suggested superiority of medication over EEG-neurofeedback in terms of both behaviour and neural activity. ${ }^{11}$ Notably, these studies scantily report whether participants learned to modulate the brain signal of interest and thus provide little insight into the neural underpinnings of these effects. To promote EEGneurofeedback as a brain-regulation therapy, researchers will need to conduct high-quality clinical trials that confirm the alleged underlying neurological mechanisms and highlight an advantage over sham neurofeedback. To justify clinical application, EEG-neurofeedback needs to perform similar to, or better than, currently accepted treatments. If research proves EEGneurofeedback effective, even if psychosocial factors rather than neurological substrates drive clinical improvement, practitioners could find ways to apply this intervention in a fashion that is both scientifically judicious and ethically acceptable. Meanwhile, unlike with EEG, nascent findings from neurofeedback with functional magnetic resonance imaging seem to pave a promising, albeit tentative, road towards the coveted holy grail of the self-regulating brain. ${ }^{12}$ 


\section{ACKNOWLEDGEMENTS}

We thank the editor as well as our colleague, Michael Lifshitz, for comments on earlier drafts of this manuscript. Dr. Amir Raz acknowledges funding from the Canada Research Chair program, Discovery and Discovery Acceleration Supplement grants from the Natural Sciences and Engineering Research Council of Canada (NSERC), Canadian Institutes of Health Research, and the Bial Foundation. Robert T. Thibault, also a Bial recipient, acknowledges an Alexander Graham Bell Canada Graduate Scholarship from NSERC. The funding sources had no involvement in reviewing the literature, writing the manuscript, or deciding to submit the paper for publication.

\section{CONTRIBUTORS}

RTT reviewed the literature, consulted with experts, and prepared the initial draft. RTT and AR prepared the final draft together. AR provided comments throughout manuscript preparation.

\section{DECLARATION OF INTEREST}

We declare no competing interests.

\section{REFERENCES}

1 Gruzelier JH. EEG-neurofeedback for optimising performance. I: A review of cognitive and affective outcome in healthy participants. Neurosci Biobehav Rev 2014; 44: 124-41.

2 Hammond DC. What is Neurofeedback: An Update. J Neurother 2011; 15: 305-36.

3 Thibault R, Lifshitz M, Birbaumer N, Raz A. Neurofeedback, Self-Regulation, and Brain Imaging : Clinical Science and Fad in the Service of Mental Disorders. Psychother 
Psychosom 2015; 84: 193-207.

4 Arnold LE, Lofthouse N, Hersch S, et al. EEG neurofeedback for ADHD: double-blind sham-controlled randomized pilot feasibility trial. J Atten Disord 2013; 17: 410-9.

5 Ramos-Murguialday A, Broetz D, Rea M, et al. Brain-machine interface in chronic stroke rehabilitation: a controlled study. Ann Neurol 2013; 74: 100-8.

6 Weimer K, Colloca L, Enck P. Placebo effects in psychiatry: Mediators and moderators. The Lancet Psychiatry 2015; 2: 246-57.

7 Arns M, Heinrich H, Strehl U. Evaluation of neurofeedback in ADHD: the long and winding road. Biol Psychol 2014; 95: 108-15.

8 Fuchs T, Birbaumer N, Lutzenberger W, Gruzelier JH, Kaiser J. Neurofeedback Treatment for Attention-Deficit / Hyperactivity Disorder in Children : A Comparison With Methylphenidate. Appl Psychophysiol Biofeedback 2003; 28.

9 Rossiter DTR, La Vaque TJ. A Comparison of EEG Biofeedback and Psychostimulants in Treating Attention Deficit/Hyperactivity Disorders. J Neurother 1995; 1: 48-59.

10 Janssen TWP, Bink M, Geladé K, van Mourik R, Maras A, Oosterlaan J. A randomized controlled trial into the effects of neurofeedback, methylphenidate, and physical activity on EEG power spectra in children with ADHD. J Child Psychol Psychiatry 2016.

DOI:10.1111/jcpp.12517.

11 Ogrim G, Hestad KA. Effects of neurofeedback versus stimulant medication in attentiondeficit/hyperactivity disorder: a randomized pilot study. J Child Adolesc Psychopharmacol 2013; 23: 448-57.

12 Thibault R, Lifshitz M, Raz A. The Self-Regulating Brain and Neurofeedback: Experimental Science and Clinical Vogue. Cortex 2016; 74: 247-61. 